\title{
Utilization of blood and components in a tertiary care hospital
}

\author{
Dushyant Singh Gaur · Gita Negi · Neena Chauhan · Anuradha Kusum · Sabina Khan · Ved Prakash Pathak
}

Received: 3 September 2008 / Accepted: 17 August 2009

(C) Indian Society of Hematology and Transfusion Medicine 2009

\begin{abstract}
Purpose Blood transfusion is an important part of patient management. Indications for blood use must be clear in the mind of ordering clinicians, to avoid its misuse and also to avoid unnecessary exposure of the patient to donor blood antigens, adverse reactions and transfusion transmissible diseases.
\end{abstract}

Methods In a retrospective pilot study, details of whole blood and components transfused were noted and correlated with the patient's diagnosis and indications for transfusion, during 1 month.

Results The blood units supplied were 720. Whole blood was the most utilized product; followed by packed red blood cells. Supply of blood was maximum to the surgical wards. The patients of trauma followed by malignancy and surgery required whole blood mostly. Anemia was the most common indication for blood products.

Conclusions Periodic review of blood component usage is very important to assess the blood utilization pattern in any hospital.

\footnotetext{
D. S. Gaur · G. Negi · N. Chauhan · A. Kusum · S. Khan ·

V. P. Pathak

Department of Pathology,

Himalayan Institute of Medical Sciences,

Jolly Grant, Dehradun,

Uttarakhand, India

D. S. Gaur $(\bowtie)$

E-mail: dugaur@yahoo.com
}

Keywords Blood units - Whole blood

Blood components $\cdot$ Blood usage $\cdot$ Periodic review of blood usage

\section{Introduction}

Blood transfusion constitutes an important part of various treatment protocols. Blood must be transfused keeping in mind certain precautions because, like drugs, blood and its components have the propensity to cause side effects such as introduction of donor antigens in the recipient, transfusion reactions or exposure to various transfusion transmissible diseases. It is very important for clinicians to be aware of these potential risks to the recipient of blood and its products. Thus the indications for ordering blood must be fully justified to avoid misuse or overuse of this precious resource. Hence, periodic review of blood component usage is essential to assess the blood utilization pattern in any hospital or health set-up.

\section{Material and methods}

This study was conducted at the blood bank of a tertiary health care centre. The number of all whole blood units and blood components supplied in 1 year was recorded and their average was calculated. A month, the data of which was closest to the average blood supply of 1 year, was chosen as a representative month. All details of the blood and component recipients in this representative month were recorded, including age, gender, address, consultant in-charge, ward/ unit where admitted, diagnosis along with indications for transfusion. History of previous and present illness, including any previous transfusions and transfusion reactions, if any, were noted. Reports of salient investigations like 
hemoglobin $(\mathrm{Hb})$, platelet count and coagulation profile, etc. were also recorded. Details of present usage of blood, including the number and type of components transfused and reactions, if any, were noted. The usage of different types of components was recorded and correlated with the patient's diagnosis and indications for transfusion.

\section{Observations}

The total number of whole blood and its products issued from our blood bank in 1 year (1st February 2007 to 31 st January 2008) was 8,549 units; with a monthly average of 712 units. The supply showed some seasonal variation, with lesser units supplied near year ends and peak observed around mid-year (Table 1). For this study, data was collected for a month (December 2007) the blood supply of which ( $\mathrm{n}=720$ units) was closest to the calculated monthly average ( $\mathrm{n}=712$ units); to account for the seasonal variation in blood demand. In that representative 1 month, the number of requests for blood products was from 428 patients, with many of these requests being for more than 1 unit. A breakup of the supply for whole blood and various components $(n=720)$ showed that whole blood $(n=308)$ was the maximum utilized product followed by packed red blood cells (PRBC), fresh frozen plasma (FFP) and then platelet concentrates (Table 1).

Supply of blood was found to be more in the surgical wards forming $62.6 \%(n=268 / 428)$ of total supply; maximum blood required for general surgery $(n=95 / 268,35.6 \%)$. Requests for supply of blood to medical wards constituted $37.4 \%(\mathrm{n}=160 / 428)$ of all demands, with highest requirement from general medicine $(\mathrm{n}=93 / 160,58.1 \%)($ Table 2$)$.
Youngest recipient of blood unit was 3-month-old male child while the oldest was 92-year-old female. Largest number of patients were in the age group 21-60 years $(\mathrm{n}=318 / 428,74.09 \%)$. Male:female ratio of the patients was $1.6: 1$.

The most common diagnosis for patients requiring blood products was trauma $(\mathrm{n}=88 / 428,20.6 \%)$; followed by malignancy $(\mathrm{n}=74 / 428,17.3 \%)$ and elective surgery $(\mathrm{n}=63 / 428,14.7 \%)$ (Table 3$)$.

Among the indications for all blood products taken together, anemia was the most common indication $(\mathrm{n}=$ $176 / 428,41.1 \%)$ followed by elective surgery $(n=123 / 428$, 28.7\%) (Table 4).

The total demands for whole blood were 211 out of 428 cases $(49.3 \%)$. The most common indication for whole blood was surgical procedures, post-traumatic, emergency as well as elective $(\mathrm{n}=113 / 211,53.56 \%)$; followed by anemia $(n=82 / 211,38.87 \%)$. Among the 104 requests for PRBCs, the indications were anemia $(n=94 / 104)$ and surgery $(n=10 / 104)$. FFP had been transfused to 75 patients. Of these, 38 patients had markedly deranged coagulation profiles; while another 30 cases had bleeding as the indication, with results of coagulation study not documented. Bleeding at the time of surgery was the indication in 7 patients. The number of requests for platelets was $38(\mathrm{n}=38 / 428,8.9 \%)$. The most common indication was thrombocytopenia due to leukemia $(\mathrm{n}=$ 11/38). Thrombocytopenia at the time of surgery was the indication in $5(\mathrm{n}=5 / 38)$ patients. Three newborn children $(n=3 / 38)$ were given platelet concentrates, the indications being septicemia with thrombocytopenia in two and preterm baby with thrombocytopenia in one case.

Table 1 Utilization of blood and blood components in one year

\begin{tabular}{|c|c|c|c|c|c|c|}
\hline S. No. & Month & Whole blood & PRBC & FFP & Platelets & Total \\
\hline 1. & February '07 & 264 & 90 & 65 & 15 & 434 \\
\hline 2. & March '07 & 340 & 200 & 110 & 54 & 704 \\
\hline 3. & April '07 & 220 & 120 & 80 & 50 & 470 \\
\hline 4. & May '07 & 357 & 250 & 170 & 90 & 867 \\
\hline 5. & June '07 & 357 & 230 & 170 & 90 & 847 \\
\hline 6. & July '07 & 311 & 155 & 105 & 45 & 616 \\
\hline 7. & August '07 & 370 & 255 & 180 & 120 & 925 \\
\hline 8. & September '07 & 340 & 210 & 150 & 120 & 820 \\
\hline 9. & October '07 & 336 & 175 & 200 & 120 & 831 \\
\hline 10. & November '07 & 276 & 170 & 210 & 70 & 726 \\
\hline 11. & December '07 & 294 & 210 & 150 & 75 & 720 \\
\hline \multirow[t]{3}{*}{12.} & January '08 & 234 & 145 & 65 & 145 & 589 \\
\hline & Total & 3,699 & 2,210 & 1,655 & 994 & 8,549 \\
\hline & Average & 308 & 184 & 138 & 83 & 712 \\
\hline
\end{tabular}

PRBC $=$ Packed red blood cell; FFP $=$ Fresh frozen plasma 
Table 2 Supply of blood to different wards $(n=428)$

\begin{tabular}{clcc}
\hline & & \multicolumn{2}{c}{ Blood units } \\
\cline { 3 - 4 } S. No. & Ward & Number & Percentage \\
\hline A & Surgical wards & 268 & 62.6 \\
1. & General surgery & 95 & 35.6 \\
2. & Orthopedics & 54 & 20.1 \\
3. & Neurosurgery & 51 & 19.0 \\
4. & Gynecology & 41 & 15.3 \\
5. & Otolaryngology & 14 & 5.2 \\
6. & Cardiothoracic & 7 & 2.6 \\
& surgery & & \\
7. & Others & 6 & 2.2 \\
B & Medical wards & 160 & 37.4 \\
1. & General medicine & 93 & 58.1 \\
2. & Nephrology & 40 & 25.0 \\
3. & Pediatrics & 24 & 15.0 \\
4. & Others & 3 & 1.9 \\
\hline
\end{tabular}

Table 3 Diagnoses of patients requiring transfusion $(n=428)$

\begin{tabular}{llcc}
\hline S. No. & Diagnosis & Number & Percentage \\
\hline 1. & Trauma & 88 & 20.6 \\
2. & Malignancy & 74 & 17.3 \\
3. & Elective surgery & 63 & 14.7 \\
4. & Hepatic disorders & 59 & 13.8 \\
5. & Renal disorders & 56 & 13.1 \\
6. & Gynecological conditions & 48 & 11.2 \\
7. & Bleeding disorders & 23 & 5.3 \\
8. & Infections & 17 & 4.0 \\
\hline & Total & 428 & 100
\end{tabular}

Drug Administration (FDA) as their use is meant to produce therapeautic benefits to the patient. Thus FDA emphasizes on the quality of blood and its products by applying pharmaceutical industry standards to the collection, testing, storage and supply of safe blood [1].

It is important for the blood bank to be able to fulfill the demands for this life-saving product and at the same time, evaluate and assess the existing trends of blood ordering. This is important to prevent misuse which may lead to shortage of blood availability and thus denial of blood supply to someone in a life-threatening situation. The importance of an internal audit and education programs, emphasizing proper selection of blood components for patients and avoiding their overuse, has been highlighted by various authors who reported a marked decline in inappropriate demands for blood products after such audits were followed by educational sessions for clinicians [2-7].

Indications for blood usage have been defined over the years. In the post-World War II era, a popular trigger for blood transfusion was a $\mathrm{Hb}$ level less that $10 \mathrm{~g} / \mathrm{dl}$ due to any cause. Presently, with advent of blood component usage for specific needs of patients, better guidelines have been suggested and put to practice globally. In many hospitals, it is now a standard practice of blood bank to manufacture different blood components from donated whole blood units and supply only components thereafter to patients $[3,4,6,8]$.

However, use of whole blood is recommended in certain situations, instead of replenishment of blood loss through components. One such situation where use of whole blood is preferentially indicated is polytrauma, as in road accident casualties. Advanced trauma life support (ATLS) guidelines recommend blood transfusion in class III and IV hypovolemia, in adults $[8,9]$. An actively bleeding case with loss of

Table 4 Indications for which blood products were issued $(n=428)$

\begin{tabular}{|c|c|c|c|c|c|c|c|}
\hline S. No. & Indication & Whole blood & PRBC & FFP & Platelets & Total & Percentage \\
\hline 1. & Anemia & 82 & 94 & - & - & 176 & 41.1 \\
\hline 2. & Surgery & 113 & 10 & - & - & 123 & 28.8 \\
\hline 3. & Bleeding & 14 & - & 37 & - & 51 & 11.9 \\
\hline 4. & Deranged PT & - & - & 38 & - & 38 & 8.9 \\
\hline 5. & Thrombocytopenia & - & - & - & 38 & 38 & 8.9 \\
\hline 6. & Exchange transfusion & 1 & - & - & & 1 & 0.2 \\
\hline \multirow[t]{2}{*}{7.} & Shock & 1 & - & - & - & 1 & 0.2 \\
\hline & Total & 211 & 104 & 75 & 38 & 428 & \\
\hline
\end{tabular}

PRBC $=$ Packed red blood cell; FFP $=$ Fresh frozen plasma; $\mathrm{PT}=$ Prothrombin time

\section{Discussion}

Internal audits form an integral part of the quality control program in a blood bank, like in any other organization. The purpose of quality assurance here is to provide safe and effective blood products to patients. Whole blood and its components are considered as drugs by the Food and more that $25 \%$ of total blood volume or an active bleeding patient who has already received 4 units of PRBC, needs to be transfused with whole blood as it not only replenishes the blood volume and oxygen-carrying capacity, it replenishes, and prevents dilution of, coagulation factors too. In our audit, we found that units issued showed a ratio of whole blood:PRBC:FFP:platelets as 3.8:2.6:2.4:1. The 
ratio of whole blood to PRBC in our study was $1.5: 1$, which was different from studies which show blood usage ratio of whole blood to PRBC as 1:3 or less. Our hospital being situated in a hilly region, on a busy highway, ends up getting large number of road accident victims. Hence, in our study, the most common diagnosis of the patients requiring blood was trauma (20.6\%), including fractures, head injuries and polytrauma. Following the ATLS guidelines, more whole blood units were demanded by our clinicians, contributing to the high whole blood:PRBC ratio observed.

The current trend of replacing the lost blood volume by components, instead of whole blood unit, often leads to situations where 1 unit of PRBC is transfused along with 1 unit each of FFP and platelet concentrate, with the aim of reconstitution of whole blood in the recipient's circulatory system. What goes unnoticed is the fact that in this process, the recipient gets transfused with three sets of foreign immunological antigens from different donors! Giving whole blood produces better replenishment of blood volume, with introduction of foreign antigens from only one donor, hence exposing the recipient to lower immunological risk $[1,7-9]$.

Decreased oxygen-carrying capacity secondary to bleeding, $\mathrm{Hb}<8 \mathrm{gm} / \mathrm{dl}$, hematocrit $<24 \%$, or symptoms of specific chronic anemia, leukemia, or dialysis, all these are indications for transfusion of PRBC units [7-9]. Similarly, platelet counts $<20,000 / \mathrm{mm}^{3}$; or a case posted for operative procedure in $<12$ hours with platelet counts $<50,000 / \mathrm{mm}^{3}$, are indications for giving platelet concentrates. Indications for FFP are activated partial thromboplastin time (aPTT) more than 60 seconds; prothrombin time (PT) more than 16 secconds or clotting factors deficiency with bleeding [8]. However, these trigger levels can be safely reduced to lower levels in certain situations, without producing any overt risk to patients. For example, in most patients, a threshold up to $10,000 /$ cu.mm is likely to be as safe as previously defined higher levels $[1,4]$. Diedrich et al. found that a prophylactic platelet level of $<10,000 / \mathrm{mm}^{3}$ in allogenic hematopoeitic progenitor cell (HPC) transplant recipients was quite safe and resulted in decreased use of platelet concentrates in these cases [5].

In cases requiring surgery, blood and components are often ordered due to anticipated rather than actual blood loss [7]. This leads to overuse of blood products and unnecessary exposure of the patient to various hematological antigens and infections. Moreover, the number of crossmatches performed for such cases, is thus usually far more than the number of units ultimately transfused. As a result, some of the units are cancelled later. This anticipatory demand increases workload on the blood bank staff, encroaching upon their time, which could be utilized for processing blood for more needy patients. Moreover, during the period when these unrequired blood units remain reserved, the real needy patients are often deprived of blood units due to low stock of unreserved units in the blood bank. An estimated
$33-62 \%$ of all transfusions given post-trauma have been found to be inappropriate [9]. Following a policy of maximum surgical blood order schedule (MSBOS), where the anticipated number of units are only grouped and typed, instead of performing complete crossmatch with various donor units, should be encouraged in the blood banks [8].

The second most common diagnosis of patients requiring blood transfusions in our study was malignancy $(\mathrm{n}=$ $74 / 428,17.3 \%$ ) (Table 3). This can be attributed to the large number of patients with malignancies coming to our hospital, as it is the only tertiary care health center of this region [10]. The requirement of blood arises more in these cases due to anemia secondary to anorexia, cancer cachexia, bone marrow suppression in cases of leukemias or chemotherapy, etc. Similar high percentage of blood utilization for patients with malignances have been reported in several studies $[6$, 7, 11, 12].

Demands related to hepatic disorders $(n=59 / 428$, $13.8 \%$ ) were responsible for most of the FFP used, for patients with deranged PT or bleeding, without documented coagulation study (Tables 3 and 4). Patients with tenal disorders requiring blood transfusion were mostly those who were on renal dialysis, attributable to the busy nephrology dialysis unit in our hospital.

In our audit, anemia was the commonest indication (41.1\%) for blood transfusion demands from medical as well as many surgical wards (Table 4). The replenishment of these demands was by whole blood to PRBC ratio of 0.87:1 (WB:PRBC = 82:94). Vishwanathan et al. also found anemia to be the top indication for RBC mass replenishment in $42 \%$ cases [7]. It is very important here to consider the trigger values of $\mathrm{Hb}$ and hematocrit for blood tranfusions in case of anemia and correlate with the clinical condition of the patient [13]. Misuse of PRBC can also be avoided in patients whose $\mathrm{Hb}$ or hematocrit can be improved by other means like diet, hematinics, etc. Another trend amongst clinicians is to often transfuse 1 or 2 units or blood to moretateto-severely anemic patients, which actually is of no benefit to these patient as it does not increase the Hb or oxygen carrying capacity significantly. At the same time, it introduces several new foreign antigens in the blood circulation of the recipient. Such inappropriate transfusions must be identified and steps should be taken to prevent them. Similarly in cases of shock, sometimes blood is transfused in class I and II, where it is not recommended according to WHO guidelines [9]. Only established hypovolemia of class II and above and showing only transient or no improvement need to be transfused blood $[8,9]$.

In the case of FFP, it is recommended to transfuse 5-6 units to correct the hemostatic defect due to clotting factor deficiency [4]. Many times only 1 or 2 units are transfused. There are many such reports available regarding inappropriate transfusion of FFP at various centers, showing $29-40 \%$ FFPs being used inappropriately [14-17]. It should in fact, be the aim of all blood banks to achieve even higher rates of 
appropriate transfusion of various blood products. Yeh et al. carried out audit on FFP usage and followed it by five sessions of education on transfusion guidelines which resulted in 30\% decrease in inappropriate FFP usage [2]. Hawkins TE instituted a system of pre-transfusion approval which resulted in 33\% decrease in units of FFP transfused [3].

The supply of blood to surgical wards was high $(\mathrm{n}=$ $268 / 428,62.6 \%)$ as compared to the medical wards ( $\mathrm{n}=$ $160 / 428,37.4 \%$ ). Similarly scenatrio has been reported by many workers [12-16]. However, studies like that of Wallis et al. have reported more supply to medical patients (62\%) as compared to $33 \%$ to surgical patients [11].

Lastly the importance of autologous transfusions should be emphasized in order to ensure availability of patient's own blood which is immunologically safest and most effective for him.

\section{Conclusion}

This pilot study provides information on blood component usage in our tertiary care hospital. It demonstrates the trend of utilization of blood and blood components and is relevant for quality management of transfusion practice, cost analyses and for planning local and regional blood donation programs. Whole blood was the maximum utilized product followed by PRBC, FFP and then platelet concentrates. Supply of blood was found to be highest in the surgical wards. The most common diagnosis of patients requiring blood was trauma followed by malignancy and surgery. Overall, anemia was the most common indication. Whole blood is used in plenty at our center as it caters to a large number of trauma cases. Periodic assessment of blood usage followed by educational sessions for clinicians is recommended to its prevent misuse or overuse, which may lead to shortage of available blood to someone in a life-threatening situation.

\section{References}

1. Hillyer CD, Silberstein LE, Ness PM, Anderson KC (Eds.) (2003) Blood Banking and Transfusion Medicine. 2nd Edition, Churchill Livingstone, New York

2. Yeh CJ, Wu CF, Hsu WT, Hsieh LL, Lin SF, Liu TC (2006) Transfusion audit of fresh frozen plasma in southern Taiwan. Vox Sang 91:270-274
3. Hawkins TE, Carter JM, Hunter PM (1994) Can mandatory pretransfusion approval programmes be improved? Transfus Med 4:45-50

4. Greeno E, McCullough J, Weisdorf D (2007) Platelet utilization and the transfusion trigger: a prospective analysis. Transfusion 47:201-205

5. Diedrich B, Remberger M, Shanwell A, Svahn BM, Ringden O (2005) A prospective randomized trial of a prophylactic platelet transfusion trigger of $10 \times 10^{9}$ per L versus $30 \times 10^{9}$ per $\mathrm{L}$ in allogenic hematopoeitic progrnitor cell transplant recipients. Transfusion 45:1064-1072

6. Zimmerman R, Buscher M, Linhardt C, Handtrack D, Zinqsem J, Weisbach V, et al. (1997) A survey of blood component use in a German University Hospital. Transfusion 37:1075-1083

7. Vishwanathan C, Jain R, Kamath M (1999) Blood utilization review in a Tertiary Care Hospital. Ind J Hemat Blood Transf $17: 26-31$

8. Harmening DM (1998) Modern Blood Banking and transfusion practices. 3rd Edition, FA Davis Company Philadelphia

9. World Health Organization (2001) The clinical use of blood in medicine, obstetrics, paediatrics, surgery \& anesthesiology, trauma \& burns. WHO Blood Transfusion Safety, Geneva

10. Gaur DS, Kishore S, Harsh M, Kusum A, Bansal R (2006) Pattern of cancers amongst patients attending Himalayan Institute of Medical Sciences, Dehradun. Indian J Pathol Microbiol 49:193-198

11. Wallis JP, Wells AW, Chapman CE (2007) Changing indications for red cell transfusion from 2000-2004 in the North of England. Transfus Med 17:315-316

12. Lim YA, Lee WG, Cho SR, Hyun BH, Sc D (2004) A study of blood usage by diagnoses in a Korean University Hospital. Vox Sang 86:54-61

13. Stanworth SJ, Cockburn HA, Boralessa H, Contreras M (2002) Which group of patients are transfused? A study of red cell usage in London and southeast England. Vox Sang 83:352-357

14. Midori K, Keiko N, Kuninori K, Yuriko N, Yoko Y (2005) Blood component usage at the emergency and critical care unit, Fukuoka University Hospital. Japanese J Transfus Med 51:430-434

15. Makroo RN, Raina V, Kumar P, Thakur UK (2007) A prospective audit of transfusion requests in a tertiary care hospital for the use of fresh frozen plasma. Asian J Transfus Sci 1:59-61

16. Pratibha R, Jayaranee S, Ramesh JC, Lopez CG, Vasanthi N (2001) An audit of fresh frozen plasma usage in a tertiary referral centre in a developing country. Malays J Pathol 23:41-46

17. Chaudhary R, Singh H, Verma A, Ray V (2005) Evaluation of fresh frozen plasma usage at a tertiary care hospital in North India. ANZ J Surg 75:573-576 